\title{
Risk Factors for Helicobacter pylori Infection and Endoscopic Reflux Esophagitis in Healthy Young Japanese Volunteers
}

\author{
Yuichiro Tanaka ${ }^{1}$, Yasuhisa Sakata ${ }^{1}$, Megumi Hara ${ }^{2}$, Hiroharu Kawakubo ${ }^{1}$, Nanae Tsuruoka ${ }^{1}$, \\ Koji Yamamoto ${ }^{1}$, Yoichiro Itoh ${ }^{1}$, Hidenori Hidaka ${ }^{1}$, Ryo Shimoda ${ }^{1}$, \\ Ryuichi Iwakiri ${ }^{1}$ and Kazuma Fujimoto ${ }^{1}$
}

\begin{abstract}
:
Objective The aim of this study was to determine the prevalence and risk factors of reflux esophagitis and Helicobacter pylori (H.pylori) infection and their interrelationship in healthy young Japanese volunteers.

Methods Between 2010 and 2016, 550 fifth-year medical students at Saga Medical School, aged 22 to 30 years, underwent upper gastrointestinal endoscopy and completed a questionnaire (frequency scale for symptoms of gastroesophageal reflux disease). H. pylori infection was determined by detecting urinary immunoglobulin $\mathrm{G}$ antibodies.

Results H. pylori antibodies were detected in 45 of the 550 subjects (8.2\%). Endoscopic reflux esophagitis was detected in 38 out of 550 (6.9\%): grade A in 37 subjects (97.3\%) and grade B in 1. Most subjects with reflux esophagitis were $H$. pylori-negative (35/37). Nodular gastritis was observed in 33.3\% (15/45) of $H$. pylori-positive subjects. The risk factors for $H$. pylori infection were drinking well water in childhood, nodular gastritis, and duodenal ulcer scars. The risk factors for endoscopic reflux esophagitis were male gender and obesity (body mass index $\geq 25$ ).

Conclusion This study describes the risk factors for $\mathrm{H}$. pylori infection and reflux esophagitis in healthy young Japanese subjects. The prevalence of reflux esophagitis was relatively high, and the infection rate of H. pylori was low compared with the aged Japanese population.
\end{abstract}

Key words: endoscopy, dyspepsia, gastroesophageal reflux, nodular gastritis, F scale

(Intern Med 56: 2979-2983, 2017)

(DOI: 10.2169/internalmedicine.8669-16)

\section{Introduction}

A recent study in Japan indicated that the Helicobacter pylori $(H$. pylori) infection rate increased with age by around $1 \%$ per year, whereas the population-wide infection rate decreased in a time-dependent manner (1). Several diseases, such as peptic ulcers, mucosa-associated lymphoid tissue lymphoma, gastric cancers and chronic gastritis, are induced by $H$. pylori infection $(2,3)$. Chronic gastritis, including nodular gastritis, has been recognized in relatively young adults $(4,5)$, but the prevalence of nodular gastritis in young Japanese adults is still unclear.

Several studies in Japan have shown that the eradication of $H$. pylori induced endoscopic reflux esophagitis, suggesting that $H$. pylori infection prevented endoscopic reflux esophagitis by decreasing gastric acid secretion (6-10). Several studies in Western countries also suggested that the eradication of $H$. pylori induced reflux esophagitis $(11,12)$. However, the precise relationship between $H$. pylori infection and endoscopic reflux esophagitis is not clear, and a direct relationship between $H$. pylori infection and reflux

${ }^{1}$ Departments of Internal Medicine and Gastrointestinal Endoscopy, Saga Medical School, Japan and ${ }^{2}$ Preventive Medicine, Saga Medical School, Japan

Received: December 7, 2016; Accepted: March 31, 2017; Advance Publication by J-STAGE: September 25, 2017

Correspondence to Dr. Kazuma Fujimoto, fujimotk@cc.saga-u.ac.jp 
Table 1. Characteristics of Healthy Young Adult Volunteers Who Underwent Upper Gastrointestinal Endoscopy in Japan $(\mathbf{n}=550)$.

\begin{tabular}{lc}
\hline Age (years) & $22.5 \pm 1.8$ \\
Gender (men:women) & $315: 235$ \\
Body mass index $\left(\mathrm{kg} / \mathrm{m}^{2}\right)$ & $21.0 \pm 3.1$ \\
Endoscopic esophagitis (Los & Angeles classification) \\
Grade 0 & $512(93.0 \%)$ \\
Grade A & $37(6.7 \%)$ \\
Grade B & $1(0.2 \%)$ \\
Grade C & 0 \\
Grade D & 0 \\
Hiatus herniation & $173(31.5 \%)$ \\
Barrett's esophagus & \\
Short segment & $58(10.5 \%)$ \\
Long segment & 0 \\
Duodenal ulcers scar & $7(1.3 \%)$ \\
Nodular gastritis & $16(2.9 \%)$ \\
Helicobacter pylori infection & $45(8.2 \%)$ \\
\hline
\end{tabular}

esophagitis in young adults has not been clearly demonstrated.

The infection route of $H$. pylori in Japan is still unknown, but several studies have suggested that transmission between family members is an important route $(5,13)$. The drinking of well water might also be a major infection route in developing countries $(6,14)$.

Several studies in Japan have indicated risk factors for endoscopic reflux esophagitis (gender, obesity, hiatus herniation, smoking, alcohol consumption), but few studies have focused on risk factors for young adults (15). The aim of the present study was to determine i) the prevalence of reflux esophagitis and $H$. pylori infection in healthy young Japanese adults and ii) the risk factors for $H$. pylori infection and endoscopic reflux esophagitis.

\section{Materials and Methods}

The present study included 550 fifth-year medical students (men/women: 315/235) in Saga Medical School who provided their informed consent to participate between 2010 and 2016. The mean age of the students was $22.5 \pm 1.8$ (range, 22-30) years old. All of the participants received upper gastrointestinal endoscopy performed by experienced endoscopists on the Japanese Board of Gastrointestinal Endoscopy. H. pylori infection was determined by the urinary antibody-coated bacteria test via the immunochromatography method (RAPIRUN ${ }^{\circledR}$; Otsuka Pharmaceutical, Tokyo, Japan) (16).

Just before an endoscopic examination, the subjects completed the frequency scale for symptoms of gastroesophageal reflux disease (FSSG) questionnaire regarding upper gastrointestinal symptoms $(17,18)$. In addition, any history of drinking well water in childhood was determined through interviews.

Endoscopic esophagitis was diagnosed as grade A, B, C or D using the Los Angeles Classification (19). The FSSG questionnaire comprises 12 questions (regarding 7 acid reflux symptoms and 5 dysmotility symptoms). Each symptom was assigned a score (never $=0$; occasionally [30\%] $=1$; sometimes $[50 \%]=2$; often $[70 \%]=3$; and always [100\%] = 4). A positive symptom was defined as a symptom for which the subject evaluated the frequency with a score of $\geq 2$ (sometimes, often or always). The seven questions regarding reflux symptoms were as follows: "Do you get heartburn?", "Do you sometimes subconsciously rub your chest with your hand?", "Do you get heartburn after meals?", "Do you have an unusual (e.g. burning) sensation in your throat?", "Do some things get stuck when you swallow?", "Do you feel a bitter liquid (acid) coming up into your throat?" and "Do you get heartburn if you bend over?" The five questions regarding acid-related dyspepsia were: "Does your stomach get bloated?", "Does your stomach ever feel heavy after meals?", "Do you feel sick after meals?", "Do you feel full while eating meals?" and "Do you burp a lot?"

This study was approved by the ethics committee of Saga Medical School. A statistical evaluation was carried out using $\chi^{2}$ tests, Student's t-tests and multiple logistic regression analyses with the odds ratios and $95 \%$ confidence intervals determined using the SPSS software program (version 22; SPSS, Tokyo, Japan). Statistical significance was established at a $\mathrm{p}$ value of $<0.05$.

\section{Results}

Table 1 shows the characteristics of the 550 subjects (315 men, 235 women) in the present study. The mean age of the subjects was $22.5 \pm 1.8$ years, and the mean body mass index (BMI) was $21.0 \pm 3.1 \mathrm{~kg} / \mathrm{m}^{2}$. Among the 550 subjects, 38 $(6.9 \%)$ had endoscopic esophagitis (grade A: 37, grade B: 1), and $173(31.5 \%)$ had hiatus herniation. The frequency of short-segmented Barrett's esophagus was relatively high (10.5\%, n=58), whereas no subjects had long-segmented Barrett's esophagus. The frequency of positive $H$. pylori infection was $8.2 \%$ (45/550). No subjects received eradication therapy for $H$. pylori infection, and none had taken gastric acid secretion inhibitors. A duodenal ulcer scar was detected in 7 subjects $(1.3 \%)$, including $3 \mathrm{H}$. pylori-positive subjects. Nodular gastritis was detected in 16 subjects (7 men, 9 women) (2.9\%), including $15 \mathrm{H}$. pylori-positive subjects, by gastrointestinal endoscopy.

Table 2 shows the risk factors for $H$. pylori infection in these subjects evaluated by a multivariate analysis. Gender and body weight were not markedly different between the $H$. pylori-positive and $H$. pylori-negative subjects. The smoking rate in both the positive and negative groups was very low, and the difference was not significant. The frequency of alcohol consumption tended to be higher in the positive subjects than in the negative ones, but not significantly so. The rates of hiatus herniation and Barrett's esophagus were not markedly different between the two positivity groups. The frequency of drinking well water in 
Table 2. Risk Factors For Helicobacter Pylori infection as Evaluated by a Multivariate Analysis.

\begin{tabular}{llllll}
\hline Factors & $\begin{array}{l}\text { H.pylori }(+) \\
\mathrm{n}=45 \\
(8.1 \%)\end{array}$ & $\begin{array}{l}\text { H.pylori }(-) \\
\mathrm{n}=505 \\
(91.8 \%)\end{array}$ & $\begin{array}{l}\text { Odds } \\
\text { ratio }\end{array}$ & 95\% CI & p value \\
\hline Gender (men:women) & $28: 17$ & $287: 218$ & 0.81 & $0.42-1.55$ & 0.52 \\
BMI (>25) & $4(8.9 \%)$ & $23(4.6 \%)$ & 2.91 & $0.88-9.64$ & 0.08 \\
Smoking & $1(2.2 \%)$ & $44(8.7 \%)$ & 0.43 & $0.54-3.33$ & 0.42 \\
Alcohol & $42(93.3 \%)$ & $438(79.6 \%)$ & 2.58 & $0.75-8.94$ & 0.14 \\
Herniation & $12(26.7 \%)$ & $161(31.9 \%)$ & 0.69 & $0.34-1.43$ & 0.32 \\
Barrett's esophagus & $3(6.7 \%)$ & $55(10.9 \%)$ & 0.62 & $0.18-2.15$ & 0.45 \\
Drinking well water & $21(46.7 \%)$ & $82(16.2 \%)$ & 4.96 & $2.59-9.50$ & $<0.01$ \\
\hline
\end{tabular}

The age is presented as the mean \pm standard deviation. H. pylori: Helicobacter pylori, BMI: body mass index $\left(\mathrm{kg} / \mathrm{m}^{2}\right)$, DU: duodenal ulcer, $95 \% \mathrm{CI}$ : confidence interval

Table 3. Risk Factors for Endoscopic Reflux Esophagitis as Evaluated by a Multivariate Analysis.

\begin{tabular}{|c|c|c|c|c|c|}
\hline Factors & $\begin{array}{l}\text { Esophagitis } \\
(+) \\
n=38 \\
(6.9 \%)\end{array}$ & $\begin{array}{l}\text { Esophagitis } \\
(-) \\
n=512 \\
(93.1 \%)\end{array}$ & $\begin{array}{l}\text { Odds } \\
\text { ratio }\end{array}$ & $95 \% \mathrm{CI}$ & $\mathrm{p}$ value \\
\hline Gender (men:women) & $34: 4$ & $281: 231$ & 0.14 & $0.05-0.43$ & $<0.01$ \\
\hline BMI (>25) & $6(15.8 \%)$ & $21(4.1 \%)$ & 5.23 & $1.76-15.52$ & $<0.01$ \\
\hline Smoking & $1(2.6 \%)$ & $25(4.9 \%)$ & 0.37 & $0.05-3.02$ & 0.36 \\
\hline Alcohol consumption & $33(89.5 \%)$ & $447(86.9 \%)$ & 1.39 & $0.48-4.05$ & 0.54 \\
\hline Herniation & $17(44.7 \%)$ & $156(30.3 \%)$ & 1.35 & $0.66-2.75$ & 0.41 \\
\hline Barrett's esophagus & $5(13.2 \%)$ & $53(10.2 \%)$ & 1.39 & $0.49-4.00$ & 0.54 \\
\hline H. pylori & $3(7.9 \%)$ & $42(8.2 \%)$ & 0.66 & $0.15-2.90$ & 0.58 \\
\hline
\end{tabular}

The age is presented as the mean \pm standard deviation. BMI: body mass index $\left(\mathrm{kg} / \mathrm{m}^{2}\right), 95 \% \mathrm{CI}$ : confidence interval, DU: duodenal ulcer, H. pylori: Helicobacter pylori

childhood was significantly higher in the $H$. pylori-positive subjects than in the $H$. pylori-negative subjects $(46.7 \%$ vs. $16.2 \%)$.

Table 3 shows the risk factors for endoscopic reflux esophagitis as evaluated by a multivariate analysis. Reflux esophagitis was more frequently seen in men than in women. Specifically, 34 out of 315 men $(10.8 \%)$ but only 4 out of 235 women $(1.7 \%)$ suffered from reflux esophagitis. High BMI was a risk factor for endoscopic reflux esophagitis. Other factors, including smoking, alcohol consumption, hiatus herniation, Barrett's esophagus and $H$. pylori infection, were not risk factors for reflux esophagitis.

The results of the FSSG questionnaire for endoscopic reflux esophagitis symptoms are shown in Table 4. The FSSG scores were not markedly different between the reflux and non-reflux groups. The rate of positive symptoms was not markedly different for any question. These results suggest that a symptomatic diagnosis with the FSSG questionnaire might not be effective for detecting reflux esophagitis in young adults.

\section{Discussion}

The present study revealed that the $H$. pylori infection rate was less than $10 \%$ in young adults in Japan, which was consistent with previous results (1). This study also indicated that the risk factors for $H$. pylori infection in young adults were drinking well water in childhood, nodular gastritis and duodenal ulcer scars shown by upper gastrointestinal endoscopy. Previous studies in Japan have suggested that a major infection route is transmission between family members-i.e. to a child from siblings, parents or grandparents $(13,20,21)$. The results of this study suggested that drinking well water in childhood might be another infection route for $H$. pylori in Japan, as observed in developing countries (14).

The prevalence of endoscopic esophagitis was not affected by $H$. pylori infection in young adults in the present study. Several previous studies in Japan showed that the prevalence of reflux esophagitis was lower in $H$. pylori-positive subjects than in $H$. pylori-negative subjects (6-10). The low infection rate in young adults as indicated in this study might be one of the reasons why $H$. pylori infection showed no influence on endoscopic esophagitis.

Previous studies have reported that nodular gastritis was diagnosed in relatively young women $(4,5)$. The present study demonstrated that the prevalence of nodular gastritis was $33 \%$ in H. pylori positive adults in their 20s in Japan, and the prevalence was not markedly different between men and women. Regarding atrophic gastritis, the present study 
Table 4. A Comparison of the Upper Gastrointestinal Symptoms Evaluated by the FSSG Questionnaire between Endoscopic Esophagitis-positive and Endoscopic Esophagitis-negative Subject.

\begin{tabular}{|c|c|c|c|c|c|}
\hline & $\begin{array}{l}\text { Esophagitis } \\
(+) \\
(n=38)\end{array}$ & $\begin{array}{l}\text { Esophagitis } \\
(-) \\
(n=512)\end{array}$ & $\begin{array}{l}\text { Odds } \\
\text { ratio }\end{array}$ & $95 \% \mathrm{CI}$ & $\mathrm{p}$ value \\
\hline Heartburn & $1(2.5 \%)$ & $17(3.3 \%)$ & 0.81 & $0.11-6.29$ & 0.84 \\
\hline Stomach gets bloated & $7(17.9 \%)$ & $79(15.4 \%)$ & 1.20 & $0.51-2.80$ & 0.68 \\
\hline Stomach feels heavy after meals & $8(20.5 \%)$ & $98(19.2 \%)$ & 1.09 & $0.48-2.43$ & 0.54 \\
\hline Subconsciously rub your chest with your hand & $3(7.6 \%)$ & $35(6.8 \%)$ & 1.13 & $0.33-3.86$ & 0.84 \\
\hline Feel sick after meals & $5(12.8 \%)$ & $50(9.8 \%)$ & 1.35 & $0.51-3.62$ & 0.55 \\
\hline Heartburn after meals & $5(12.8 \%)$ & $40(7.8 \%)$ & 1.72 & $0.64-4.66$ & 0.275 \\
\hline Unusual sensation in your throat & $2(5.1 \%)$ & $18(3.5 \%)$ & 1.48 & $0.33-6.61$ & 0.61 \\
\hline Feel full while eating meals & $2(5.1 \%)$ & $53(10.4 \%)$ & 0.47 & $0.11-1.99$ & 0.29 \\
\hline Get stuck when you swallow & $0(0 \%)$ & $14(2.75 \%)$ & - & - & 0.35 \\
\hline Bitter liquid coming up into your throat & $6(15.4 \%)$ & $34(6.67 \%)$ & 2.55 & $0.10-6.50$ & 0.054 \\
\hline Burp a lot & $6(15.4 \%)$ & $65(12.7 \%)$ & 1.25 & $0.50-3.09$ & 0.64 \\
\hline Heartburn if you bend over & $2(5.13 \%)$ & $10(2.0 \%)$ & 2.70 & $0.57-12.8$ & 0.19 \\
\hline Number of subjects with FSSG scores of more than 8 & $13(33.3 \%)$ & $114(22.3 \%)$ & 1.73 & $0.87-3.49$ & 0.117 \\
\hline
\end{tabular}

$95 \%$ CI: confidence interval. A positive symptom was defined as one for which the subject evaluated the frequency with a score of $\geq 2$.

did not evaluate the presence of atrophic gastritis with the upper gastrointestinal endoscopy.

The risk factors for endoscopic reflux esophagitis were male gender and obesity (BMI>25). Our previous study indicated that obesity was a risk factor for reflux esophagitis in men in their 30 s and 40 s $(22,23)$, and several studies in Japan have shown that obesity exacerbates endoscopic reflux esophagitis $(24,25)$. These data are compatible with the present results for endoscopic reflux esophagitis in adults in their 20s. Why the prevalence of reflux esophagitis was high in men in the present study was not clear, although obesity and alcohol drinking are more popular in men than in women in Japan. However, in the present study, endoscopic esophagitis was not affected by alcohol consumption, smoking or hiatus herniation. In addition, the smoking rate was very low in the present study $(4.7 \%)$ compared with the Japanese average rate $(19.3 \%)$, possibly due to that the subjects in the present study were medical students and therefore more conscientious of the effects of smoking. Alcohol consumption was evaluated based on the frequency of drinking, not by the quantity consumed. The rate of hiatus hernia was also low in the present study.

Clinical symptoms were evaluated by the FSSG questionnaire $(17,26-29)$ before the endoscopic examination. Regarding the scores for the 12 evaluated symptoms, there were no marked differences between the normal subjects and those with endoscopic esophagitis. All of the subjects with reflux esophagitis in the present study had mild symptoms of grade A or B under the Los Angeles classification, which might be one of reasons why they did not complain of clinical symptoms.

H. pylori infection was only detected by the presence of urinary IgG antibodies with immunochromatography and not evaluated by other methods, such as the rapid urease test or the urea breath test. The sensitivity of the immunochroma- tography method is lower than with other methods, which might be one reason why there were several $H$. pylorinegative subjects among those with duodenal ulcer scars or nodular gastritis. This observation warrants further explanation.

In summary, the present study of healthy young adult volunteers who received upper gastrointestinal endoscopy revealed the following: i) the infection rate of $H$. pylori was $8.2 \%$, ii) the prevalence of endoscopic reflux esophagitis was $6.9 \%$, iii) $H$. pylori infection had no influence on the prevalence of endoscopic reflux esophagitis, iv) nodular gastritis accounted for $33.3 \%$ of $H$. pylori infection, v) the risk factors for $H$. pylori infection were drinking well water in childhood, nodular gastritis and duodenal ulcer scars and vi) the risk factors for endoscopic reflux esophagitis were male gender and obesity (BMI>25).

The authors state that they have no Conflict of Interest (COI).

\section{References}

1. Hirayama Y, Kawai T, Otani J, Kawakami K, Harada Y. Prevalence of Helicobacter pylori infection with healthy subject in Japan. J Gastroenterol Hepatol 29: 16-19, 2014.

2. Furuta T, Delchier JC. Helicobacter pylori and non-malignant diseases. Helicobacter 14: 29-35, 2009.

3. Matsuo T, Ito M, Tanata S, Yoshihara M, Chayama K. Low prevalence of Helicobacter pylori-negative gastric cancer among Japanese. Helicobacter 16: 415-419, 2011.

4. Miyamoto M, Haruma K, Yoshihara M, et al. Nodular gastritis in adults is caused by Helicobacter pylori infection. Dig Dis Sci 48: 968-975, 2003.

5. Shiotani A, Kamada T, Kumamoto M, et al. Nodular gastritis in Japanese young adults: endoscopic and histological observations. Gastroenterology 42: 610-615, 2007.

6. Koike T, Ohara S, Sekine H, et al. Helicobacter pylori infection prevents erosive reflux esophagitis by decreasing gastric acid se- 
cretion. Gut 49: 330-334, 2001.

7. Kawai T, Yamamoto K, Fukuzawa M, et al. Helicobacter pylori infection and reflux esophagitis in young and middle-aged Japanese subjects. J Gastroenterol Hepatol 25: 80-85, 2010.

8. Iijima K, Koike T, Shimosegawa T. Reflux esophagitis triggered after Helicobacter pylori eradication: a noteworthy demerit of eradication therapy among the Japanese? Front Microbiol 9: 566, 2015.

9. Sugimoto M, Uotani T, Ichikawa H, Andoh A, Furuta T. Gastroesophageal reflux disease in time covering eradication for all patients infected with Helicobacter pylori in Japan. Digestion 93: 24-31, 2016.

10. Fujiwara Y, Arakawa T. Epidemiology and clinical characteristics of GERD in the Japanese population. J Gastroenterol 4: 518-534, 2009.

11. Yachida S, Saito D, Kozu T, et al. Endoscopically demonstrable esophageal changes after Helicobacter pylori eradication in patients with gastric disease. J Gastroenterol Hepatol 16: 1346-1352, 2001.

12. Labenz J. Current role of acid suppressants in Helicobacter pylori eradication therapy. Best Pract Res Clin Gastroenterol 15: 413431, 2001.

13. Okuda M, Tachikawa $T$, Maekawa $K$, Fukuda $Y$. Transmission route of H.pylori. Nihon Rinsho 71: 1339-1345, 2013 (in Japanese, Abstract in English).

14. Rolle-Kampczyk UE, Fritz U, Lehmann I, Richter M, Herbarth O. Well water - one source of Helicobacter pylori colonization. Int J Hyg Environ Health 207: 363-368, 2004.

15. Takashima T, Iwakiri R, Sakata Y, et al. Endoscopic reflux esophagitis and Helicobacter pylori infection in young healthy Japanese volunteers. Digestion 86: 55-56, 2012.

16. Murakami K, Kamada T, Ishikawa $\mathrm{H}$, et al. An evaluation of the performance of a novel stick-type kit for rapid detection of Helicobacter pylori antibodies in urine. Clin Lab 57: 481-487, 2011.

17. Kusano M, Shimoyama Y, Sugimoto S, et al. Development and evaluation of FSSG: frequency scale for the symptoms of GERD. J Gastroenterol 39: 888-891, 2004.

18. Komatsu-Tanaka M, Iwakiri R, Fujimoto K, et al. Clinical symptoms of FSSG in gastroesophageal reflux disease are critical for PPI treatment: Japanese multi-centers with 185 patients. Dig Endosc 24: 407-411, 2012.

19. Armstrong D, Bennett JR, Blum AL, et al. The endoscopic assess- ment of esophagitis: a progress report on observer agreement. Gastroenterology 111: 85-92, 1996.

20. Okuda M, Osaki T, Lin Y, et al. Low prevalence and incidence of Helicobacter pylori infection in children: a population-based study in Japan. Helicobacter 20: 133-138, 2015.

21. Urita Y, Watanabe T, Kawagoe N, et al. Role of infected grandmothers in transmission of Helicobacter pylori to children in a Japanese rural town. J Paediatr Child Health 49: 394-398, 2013.

22. Fujimoto K, Iwakiri R, Okamoto $K$, et al. Characteristics of gastroesophageal reflux disease in Japan: increased prevalence in elderly women. J Gastroenterol 38: 3-6, 2003.

23. Furukawa N, Iwakiri R, Koyama $T$, et al. Proportion of reflux esophagitis in 6010 Japanese adults: prospective evaluation by endoscopy. J Gastroenterol 24: 441-444, 1999.

24. Moki F, Kusano M, Mizuide M, et al. Association between reflux oesophagitis and features of the metabolic syndrome in Japan. Aliment Pharmacol Ther 26: 1069-1075, 2007.

25. Iwakiri K, Kinoshita Y, Habu Y, et al. Evidence-based clinical practice guidelines for gastroesophageal reflux disease 2015. J Gastroenterol 51: 751-767, 2016.

26. Danjo A, Yamaguchi K, Fujimoto K, et al. Comparison of endoscopic findings with symptom assessment systems (FSSG and QUEST) for gastroesophageal reflux disease in Japanese centres. J Gastroenterol Hepatol 24: 633-638, 2009.

27. Akutagawa K, Iwakiri R, Hara M, et al. Risk factors for low response to proton-pump inhibitor treatment in reflux esophagitis and non-erosive reflux disease evaluated by the frequency scale for the symptoms of gastroesophageal reflux disease. Esophagus 12: 225-232, 2015.

28. Kawakubo H, Tanaka Y, Tsuruoka N, et al. Upper gastrointestinal symptoms are more frequent in female than male young healthy Japanese volunteers as evaluated by questionnaire. J Neurogastroenterol Motil 22: 248-253, 2016.

29. Takeshita R, Sakata Y, Hara M, et al. Higher frequency of reflux symptoms and acid -related dyspepsia in women than men regardless of endoscopic esophagitis: analysis of 3,505 Japanese subjects undergoing medical health checkups. Digestion 93: 266-271, 2016.

The Internal Medicine is an Open Access article distributed under the Creative Commons Attribution-NonCommercial-NoDerivatives 4.0 International License. To view the details of this license, please visit (https://creativecommons.org/licenses/ by-nc-nd/4.0/).

(C) 2017 The Japanese Society of Internal Medicine

Intern Med 56: 2979-2983, 2017 